\title{
A High-Level Petri Net Based Decision Support System for Real-Time Scheduling and Control of Flexible Manufacturing Systems: An Object-Oriented Approach
}

\author{
Gonca Tuncel and Gunhan Mirac Bayhan \\ Department of Industrial Engineering, \\ University of Dokuz Eylul, \\ 35100 Bornova-Izmir, TURKEY \\ \{gonca.tuncel, mirac.bayhan\}@deu.edu.tr
}

\begin{abstract}
Petri nets (PNs) are powerful tools for modeling and analysis of discrete event dynamic systems. The graphical nature and mathematical foundation make Petri net based methods appealing for a wide variety of areas including real-time scheduling and control of flexible manufacturing systems (FMSs). This study attempts to propose an object-oriented approach for modeling and analysis of shop floor scheduling and control problem in FMSs using high-level PNs. In this approach, firstly, object modeling diagram of the system is constructed and a heuristic rule-base is developed to solve the resource contention problem, then the dynamic behavior of the system is formulated by high-level PNs. The methodology is illustrated by an FMS.
\end{abstract}

\section{Introduction}

Due to high complexity of flexible manufacturing systems, some mathematical programming related methods such as integer programming, linear programming, and dynamic programming often lack to describe the practical constraints of complex scheduling problems. On the other hand, classical scheduling techniques such as branch-and bound and neighborhood search techniques cause substantial increase in state enumeration, and thus computation time grows exponentially as the problem size increases. Recently, either existing techniques are improved or new scheduling approaches are developed to deal with the practical constraints of real-world scheduling problems. These methods include simulation-based approaches, expert systems, PNs based methods, AI-based search techniques, and hybrid methods [1]. Although the first applications of PNs were in the field of communication protocols and computer systems, they have been extended and applied to the broader range of problems including the scheduling of production systems by means of their modeling capabilities and formulation advantages. A significant advantage of PNs is its representation capability. PNs can be explicitly and concisely model concurrent, asynchronous 
activities, multi-layer resource sharing, routing flexibility, limited buffers, and precedence constraints [2], [3]. However, modeling and analysis of complex or large size systems require too much effort, since considerable number of states and transition requirements cause state explosion problem. Furthermore, PNs are system dependent and lack some features like modularity, maintability, and reusability, which are the properties of Object-Oriented approach [4]. Therefore, recently, there has been a growing interest in merging PNs and Objectoriented approach to combine graphical representation and mathematical foundation of PNs with the modularity, reusability, and maintability features of Object-orientation. Here, we present a PN based Decision Support System (DSS) for real-time shop floor scheduling and control of FMSs. High-level PNs and Object Oriented Design (OOD) approach were used for system modeling, and a heuristic rule (knowledge) based approach was employed for scheduling/dispatching in control logic. The presented DSS helps managers to take control decisions effectively and efficiently by considering the current status of the shop floor. The proposed methodology is illustrated on an example FMS. Many researchers have employed PNs for scheduling and control of manufacturing systems [5], [6], [7], [8]. However, Petri-net based dynamic scheduling of FMSs dealing with production of several product types with flexible routes, setup times, material handling system, and operator constraints has not been given much attention. In Section 2, modeling methodology is given. The paper is concluded by Section 3 .

\section{Modeling Methodology for Real-Time Shop Floor Scheduling and Control Problem of FMSs}

FMSs are characterized by concurrency, resource sharing, routing flexibility, limited buffer sizes, and variable lot sizes. For interaction activities, and the coordination of individual units, a descriptive and dynamic modeling tool is required to model in detail the concurrency, and synchronization with respect to time [1]. The units in this system can be considered as objects interacting with each other, and they can be added, removed or modified. Therefore, OOD concepts can be employed to design of shop floor scheduling and control systems in FMSs, and PNs can be used to model dynamic behavior of the system. The properties and behavior of the objects were modeled by the data/attribute and methods /operations. OOD methodology used for development of the DSS is similar to those given in [9], [10].

\subsection{Illustration of the Methodology with an FMS with Alternative Operations and Setup Times}

An FMS with alternative operations and setup times is employed to explain the modeling methodology of the proposed decision support system for real-time shop floor scheduling and control problem. The physical layout of the system considered is shown in Figure 1. The system consists of a loading and unloading 


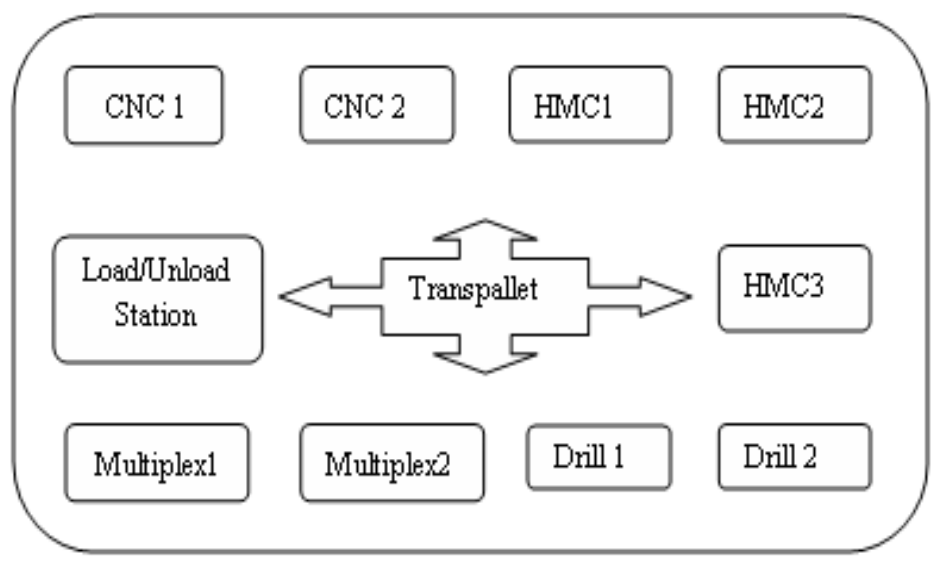

Fig. 1. Layout of the FMS

station, 9 workstations (CNC1, CNC2, Multiplex1, Multiplex 2, HMC1, HMC2, HMC3, Drill 1, and Drill 2), a material-handling system, and several operators. Each workstation has one machine with input and output buffers with limited capacity. Each machine can only process a part at a time, and once a part is processed the machine setup is needed to process a different part type. Each workstation is allowed to contain two pallets at most at a time. The FMS is designed to produce a variety of products simultaneously. Each product has alternative routes for some operations (i.e. two or more machines are enable to perform same operations). The operational policy is under push paradigm. Each job has a best operation sequence that determines the order in which resources must be assigned to the job. But the efficient utilization of resources on real time basis requires a real time resource allocation policy to assign resources to jobs as they advance through the system. Since each machine is capable of processing a variety of part types and each part has to visit a number of machines, there is often a conflict when more than one part is contending for the same machine or material handling system. The problem is the allocation of resources to a set of tasks, that is, determination of the best route of each task in the system according to the current shop-floor condition (due dates, release dates, order quantities, tardiness penalties, inventory levels, and setup status).

Object Modeling of the System. The object modeling technique (OMT) that is the most widely used OOD methodology is employed to describe and analyze the object classes. It divides the system considered into object classes, and it is used to model the static relations among FMS objects by the class structure. Each individual FMS object is derived as an instance of CPN class module. The behavior of the objects is described by operations associated with object classes. Figure 2 shows the OMT diagram corresponding to the DSS. This diagram captures the relevant properties of the FMS objects and their functions 


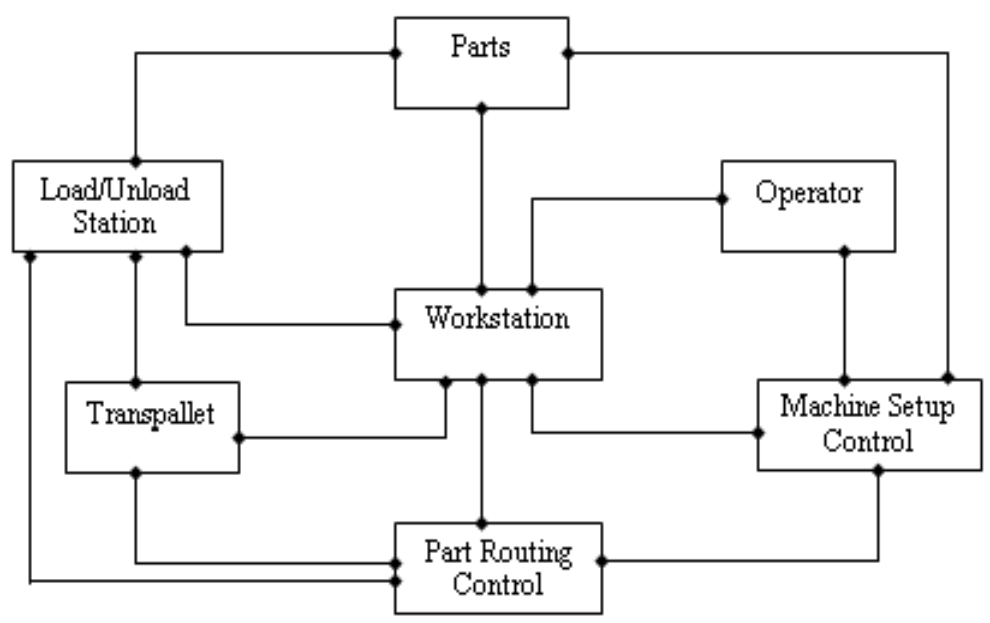

Fig. 2. OMT Diagram of the FMS

in the DSS. The links between objects with black dots at each end represent the associations. Individual classes of FMS objects are defined as follows:

Load and Unload Station Class: raw parts with the attributes such as products type, due date, and process plan, are set up on pallets and stored to the load storage buffer, and a route request is sent to the Scheduler Class for the pallet stored. When information on next destination for a pallet is received from the Scheduler Class, the pallet is moved to the active buffer, loaded to the transpallet, and sent to the destination workstation. If all operations of the parts on a pallet are completed, the pallet returns to the load/unload (L/UL) station and it is transferred to unload storage buffer. Then the finished products are unloaded from the pallet. Thus, pallets become available and are sent to the free pallet buffer to be used again.

Workstation Class: parts are processed at machining stations. Once a transpallet arrives at a workstation, the pallet is moved to the input buffer of the workstation and the transpallet becomes free. When machining station is idle, the pallet goes from the input buffer to the machining buffer, and one operator request is sent to the Operator Object. Machine setup control is also performed in WS class. If the machine operates the same part type with the previous part type, it doesn't need a setup operation. When all the parts on the pallet are processed, the pallet is sent to the output buffer of the workstation, and a routing request is sent to the Part Routing Control object. When a routing request is replied for a pallet, it is transferred to the active buffer, and then sent to the destination station by transpallet. Part transport class: parts are transported by transpallet between stations. The Part Transport Object accepts a transport request from the Scheduler Class, and forwards a transpallet to the 
station, which required it. Thus ready pallet is loaded to the transpallet, and transferred to the destination station.

Operator class: machine setup operation, loading and unloading operations are performed by operators. Operators working at workstations are represented by Operator Class.

Scheduler class: part flow between stations are controlled and managed by Scheduler Class. Part Routing Control Object is an instance of Scheduler Class. In a manufacturing system, concurrent flow of parts competing for sharing limited resources causes resource contention problem. Resource sharing in any manufacturing system often leads to conflicts. The system controller must be capable of resolving these conflicts effectively. From the standpoint of the PNs, resource sharing increases the complexity in scheduling. The proposed DSS use a heuristic rule based approach to solve resource contention problems and to determine the best route(s) of the parts, which have routing flexibility. The following heuristics are used to solve the resource contention problem:

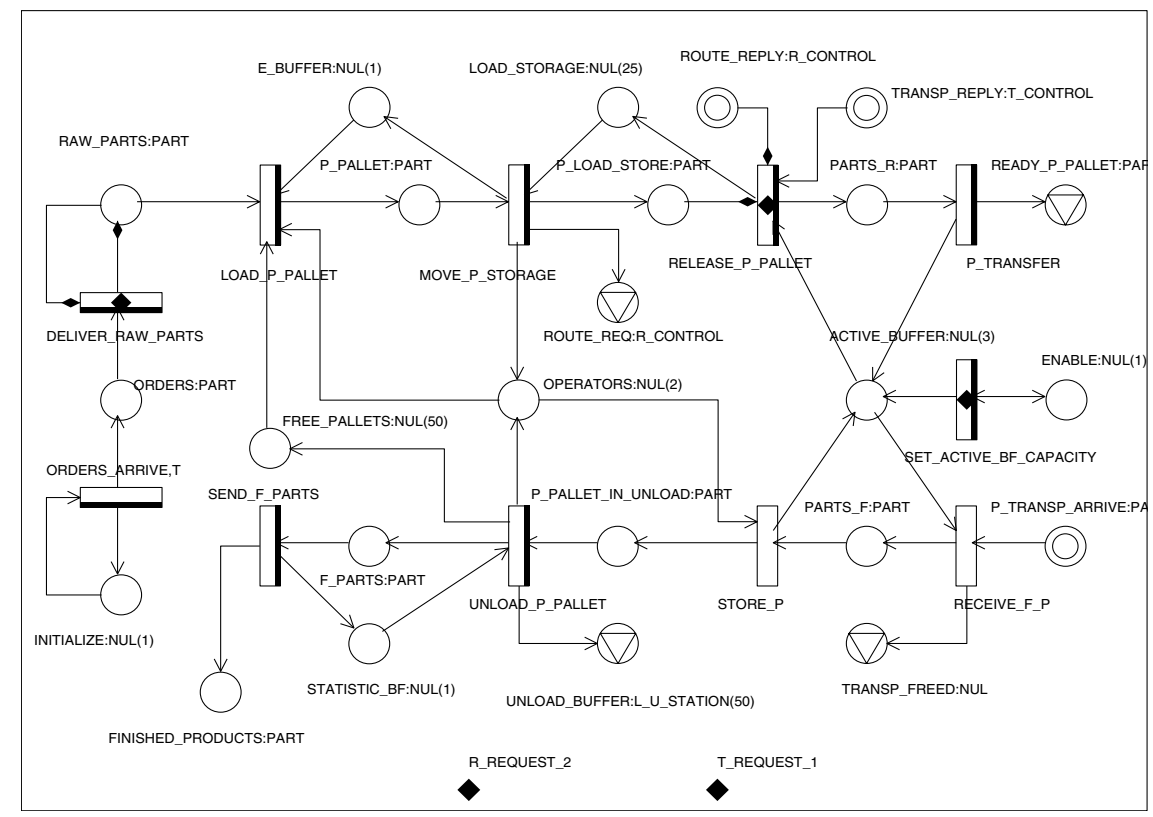

Fig. 3. CPN Model of Load / Unload Station Class

- Finished products have priority for transportation.

- Semi-finished or raw parts are ranked according to their next operation number (highest is first), or due dates (lowest is first) for the parts with the identical operation number.

- Alternative machines are ranked according to their operation time (shortest is first), and the pallet is routed to the available machine among the alternative machines which is ranked first and doesn't require setup operation. 
- If there is no available machine, which doesn't need setup operation, the route request is accepted regarding the order's critical ratio.

- After all the route requests are checked under the current system status, the route requests, which are not critical, are reevaluated to be routed.

CPN Models of FMS Objects. Dynamic behavior/control logic of the FMS is formulated by constructing high-level PN model of the system based on the static relations of the OMT model. For this purpose, each CPN class model is first constructed to capture concurrency and synchronization of the system. Internal places and transitions are used to model the operations and dynamic behavior inside the Object Class, and input and output places are used to model the interface of the objects. Then all the related PN models of classes are connected through the input and output places to obtain the complete model and the control logic of the system. In Figures through 3-7, the CPN class models are displayed.

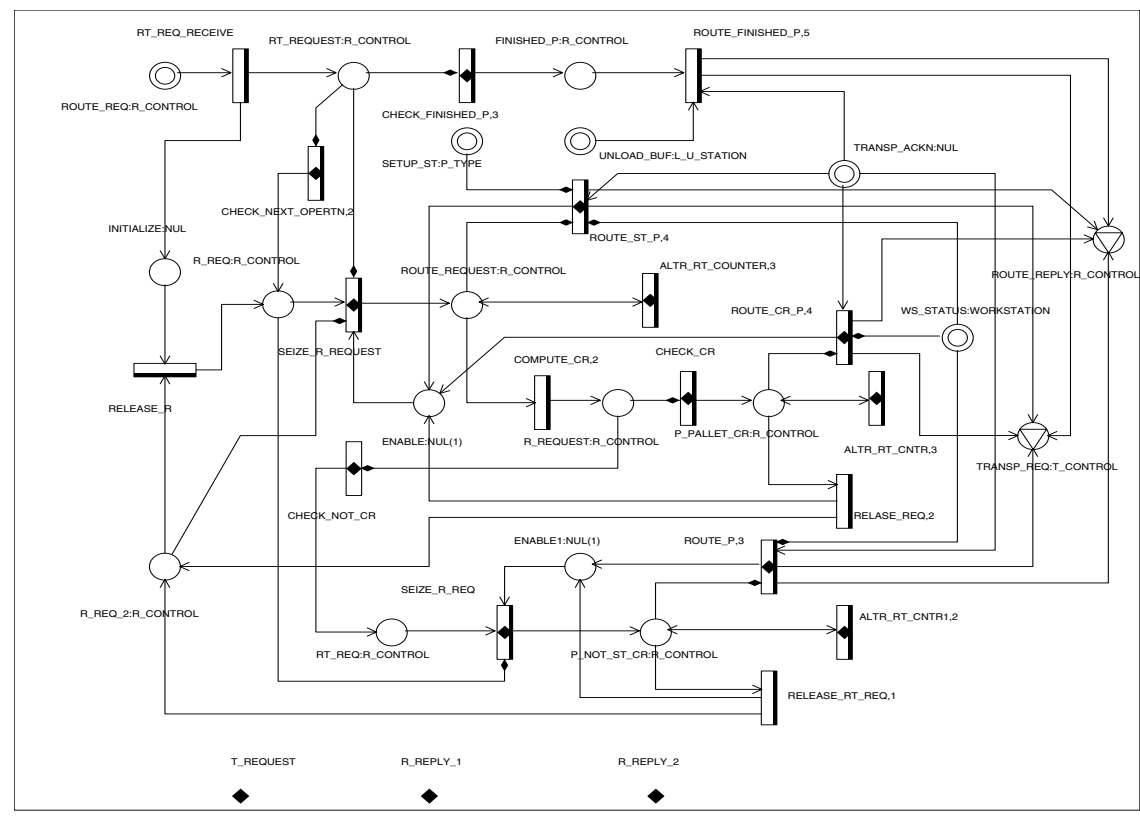

Fig. 4. CPN Model of Scheduler Class

Since the standard PNs are insufficient to model the complex and large size systems, they have been extended to High-level PNs, which allow arbitrary complex data types for tokens. Thus, transitions and places can be constructed by using a special programming language. In this study, Artifex which is a modeling and simulation environment supporting the design of discrete event systems is employed to model $\mathrm{CPN}$ classes by using a high level language $\mathrm{C} / \mathrm{C}++$. 


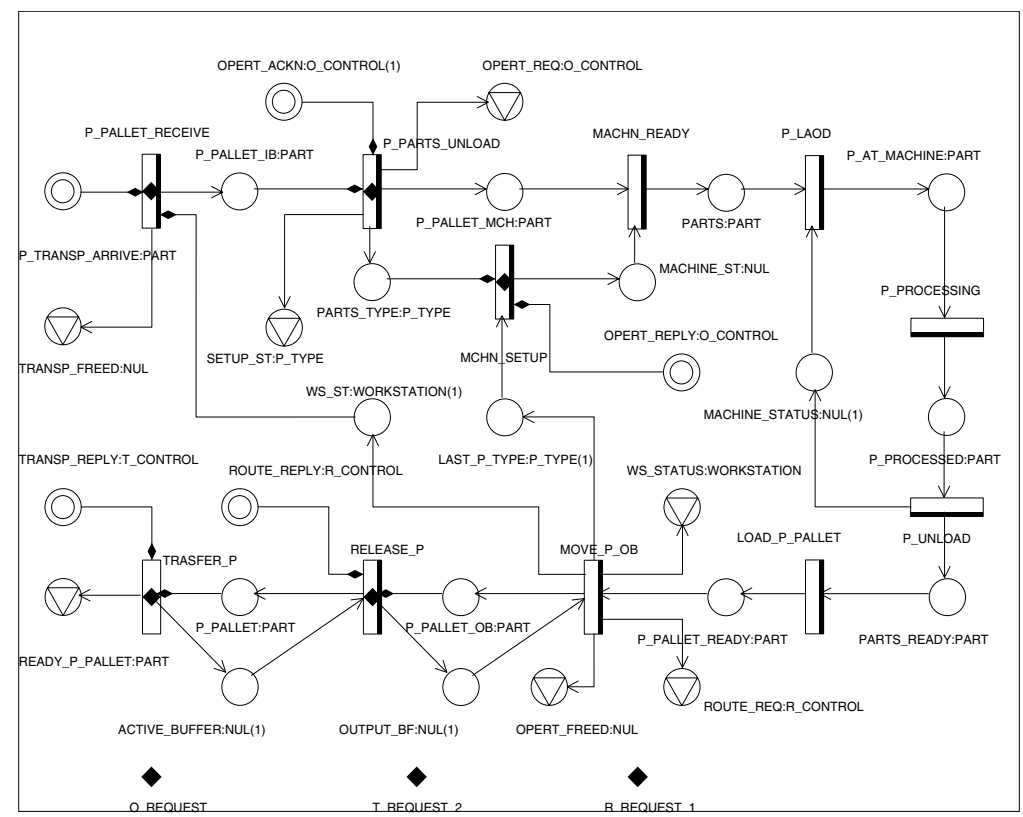

Fig. 5. CPN Model of Workstation Class

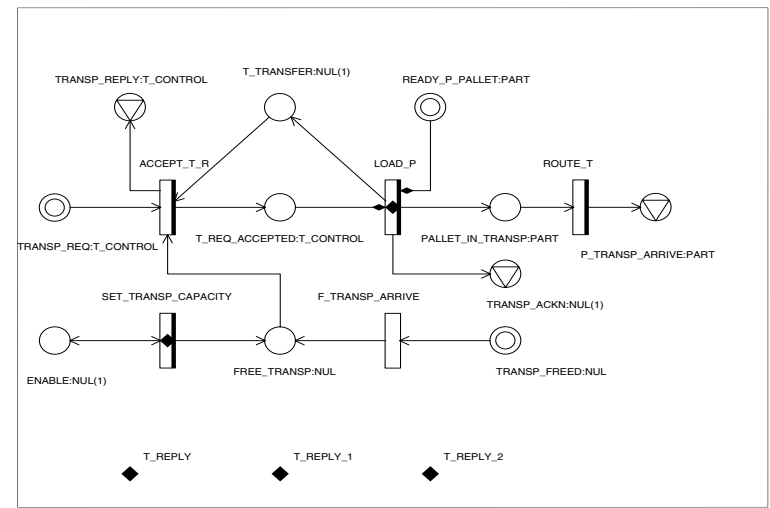

Fig. 6. CPN Model of Part Transport Class

\section{Conclusion}

In recent years, there has been a growing interest in applying $\mathrm{PN}$ theory for scheduling of production systems by means of their modeling capabilities and formulating advantages. In this study, a PN based DSS for shop floor schedul- 


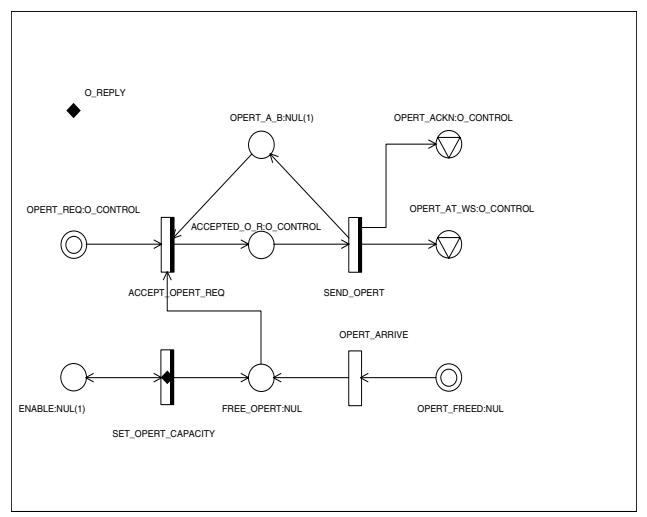

Fig. 7. CPN Model of Operator Class

ing and control of FMSs is presented. In the modeling process, object-oriented approach is used. The developed rule-based DSS aims to solve the problem of resource contention problem and to determine the best route(s) of the parts, which have routing flexibility. Decisions are taken on real-time basis by checking product types, due dates, alternative process plans, next possible destination resource, setup status, and resource utilizations rates. The DSS takes a global view of the system state before making decision about resource assignment, and proposes a dynamic approach to solve the conflict problems. It has adaptive and autonomous ability for obtaining intelligent control, and can be used for different production systems by only changing some system parameters (i.e. number of operators and stations, types of products, and process plan information) in Object Classes. Performance analysis can be performed under different system configurations.

\section{References}

1. Lin, J.T., Lee, C.C.: Petri net-based integrated control and scheduling scheme for flexible manufacturing cells. Computer Integ. Manuf.Systems, 10 (1997)109-122.

2. Murata, T.: Petri Nets: Properties, Analysis and Applications. Proceedings of the IEEE, vol.77/4 (1989) 541-580.

3. Zurawski, R., Zhou, M.C.: Petri Nets and Industrial Applications: A Tutorial. IEEE Transactions on Industrial Electronics, 4 (1994) 567-582.

4. Wang, L.: Object-oriented Petri nets for modelling and analysis of automated manufacturing systems. Computer Integ. Manuf. Systems, 26 (1996) 111-125.

5. Hatano, I., Yamagata, K., Tamura, H.: Modeling and on-line scheduling of flexible manufacturing systems using stochastic Petri nets. IEEE Trans. Software Eng., 17 (1991) 126-133.

6. Lee, D.Y., DiCesare, F.: Scheduling flexible manufacturing systems using Petri nets and heuristic search. IEEE Trans. Robot. Automat., 10 (1994) 123-133. 
7. Chetty, O.V.K., Gnanasekaran, O.C.: Modelling, simulation and scheduling of flexible assembly systems with coloured petri nets. Int. J. Adv. Manufacturing Technology, 11 (1996) 430-438.

8. Jain, P.K.: Solving resource contention problem in FMS using Petri nets and a rule-based approach. Int. J. of Production Research, 39 (2001) 785-808.

9. Chen, J., Chen, F.F.: Performance modelling and evaluation of dynamic tool allocation in flexible manufacturing systems using coloured Petri nets: An object-oriented approach. Int. J. of Adv. Manufacturing Technology, 21 (2003) 98-109.

10. Venkatesh, K., Zhou, M.C.: Object-oriented design of FMS control software based on object modeling techniques diagrams and Petri nets. J. of Manufacturing Systems, 17 (1998) 118-136. 